This is a preprint of an article accepted for publication in Electronic Notes in Discrete Mathematics (C) 2009 (copyright owner as specified in the journal). 


\title{
Wilson-Schreiber Colourings of Cubic Graphs
}

\author{
Mike J. Grannell ${ }^{3}$ Terry S. Griggs ${ }^{4}$ \\ Department of Mathematics and Statistics \\ The Open University \\ Milton Keynes MK' 6AA, U. K. \\ Edita Máčajová ${ }^{1,5}$ Martin Škoviera ${ }^{2,6}$ \\ Department of Computer Science \\ Comenius University \\ 84248 Bratislava, Slovakia
}

\begin{abstract}
An $\mathcal{S}$-colouring of a cubic graph $G$ is an edge-colouring of $G$ by points of a Steiner triple system $\mathcal{S}$ such that the colours of any three edges meeting at a vertex form a block of $\mathcal{S}$. In this note we present an infinite family of point-intransitive Steiner triple systems $\mathcal{S}$ such that (1) every simple cubic graph is $\mathcal{S}$-colourable and (2) no proper subsystem of $\mathcal{S}$ has the same property. Only one point-intransitive system satisfying (1) and (2) was previously known.
\end{abstract}

Keywords: Cubic graph, edge-colouring, Steiner triple system.

\footnotetext{
1 Supported in part by APVV, Project 0111-07

2 Supported in part by VEGA, Grant 1/0634/09

3 Email: m.j.grannell@open.ac.uk

4 Email: t.s.griggs@open.ac.uk

5 Email: macajova@dcs.fmph.uniba.sk

6 Email: skoviera@dcs.fmph.uniba.sk
} 


\section{Introduction}

A Steiner triple system $\mathcal{S}=(V, B)$ of order $v$ consists of a set $V$ of $v$ elements, called points, and a collection $B$ of 3 -element subsets of $V$, called blocks, such that every 2-element subset of $V$ is contained in exactly one block. It is well known that a Steiner triple system of order $v$ exists if and only if $v \equiv 1$ or 3 $(\bmod 6)$ (see Kirkman [5]).

Given a Steiner triple system $\mathcal{S}$, an $\mathcal{S}$-colouring of a cubic graph $G$ is an edge-colouring of $G$ by points of $\mathcal{S}$ such that the colours of any three edges meeting at a vertex form a block of $\mathcal{S}$. This kind of colouring was introduced by Archdeacon [1] in 1986 and later studied by a number of authors (for example, see $[2,4,6,8])$. Interesting connections between Steiner colourings and several difficult conjectures, such as the cycle double cover conjecture and the Fulkerson conjecture, are discussed in $[1,7,8]$.

One of the questions that naturally arise in this area is whether a given Steiner triple system $\mathcal{S}$ is universal, that is, whether every simple cubic graph admits an $\mathcal{S}$-colouring. Somewhat surprisingly, the best known geometric examples of Steiner triple systems, the projective systems $P G(n, 2)$ and the affine systems $A G(n, 3)$, include no universal member [4]. The first universal Steiner triple system (of order 381) was found by Grannell et al. [2]. Pál and Škoviera [9] improved this result by identifying a subsystem of the previous system of order 21 that is also universal. Further significant progress was made by Král' et al. [6] who proved that every non-trivial point-transitive Steiner triple system that is neither projective nor affine is universal. In particular, the smallest order of a universal system is 13 . In contrast, very little is known about colourings by point-intransitive Steiner triple systems. In fact, only one universal point-intransitive system is currently known [6].

In this note we describe an infinite family of point-intransitive universal Steiner triple systems based on the Wilson-Schreiber construction $[10,11]$. The smallest member of the family has order 15. Infinitely many of these systems are minimally universal, that is, they do not contain a proper universal subsystem. A detailed discussion and proofs will appear in a further paper [3].

\section{Wilson-Schreiber Systems and Colourings}

Let $A$ be an Abelian group of order $n$, written additively. We construct a Steiner triple system $\mathcal{S}$ of order $v=n+2$ whose points are the elements of $A$ and two additional points $\alpha$ and $\beta$. The construction applies only when, for every prime divisor $p$ of $n$, the order of $-2(\bmod p)$ is even; we call such 
a group admissible. Since $v$ is the order of a Steiner triple system, we have $v \equiv 1$ or $3(\bmod 6)$, so $n \equiv 1$ or $5(\bmod 6)$, and therefore neither 2 nor 3 divides $n$.

Let us list all unordered triples $\langle a, b, c\rangle$ of elements of $A$ with $a+b+c=0$ and repetitions allowed. For each triple $\langle a, b, c\rangle$ with pairwise distinct entries we include the set $\{a, b, c\}$ as a block of $\mathcal{S}$. The triples of the form $\langle a, a,-2 a\rangle$ where $a \in A-0$ can be partitioned into orbits under the action of the mapping $z \mapsto-2 z, z \in A$. Since $A$ is admissible, the number of triples in each orbit is even. Pick one of the orbits and replace the repeated element in each triple by $\alpha$ and $\beta$ alternately along the orbit. Process each orbit similarly, and include all sets $\{\alpha, a,-2 a\}$ and $\{\beta, b,-2 b\}$ obtained in this way as blocks of $\mathcal{S}$. Finally, replace the triple $\langle 0,0,0\rangle$ with $\{0, \alpha, \beta\}$ and include it in $\mathcal{S}$.

It is easy to see that, with the above collection of blocks, $\mathcal{S}$ is a Steiner triple system. Since there exist infinitely many primes $p$ such that -2 has even order $(\bmod p)$, there are infinitely many such Wilson-Schreiber systems. Furthermore, it can be shown that the systems constructed from the prime groups $\mathbb{Z}_{p}$ do not contain any non-trivial proper subsystem [3].

Every Wilson-Schreiber system $\mathcal{S}$ constructed from an admissible group $A$ of order greater than 9 is point-intransitive, that is, it contains two points that cannot be mapped onto each other by an automorphism of the system. This follows from the fact that the number of mitres having 0 as an apex differs from the number having $x \in A-0$ as an apex, where a mitre is partial subsystem of $\mathcal{S}$ having the form $\{\{a, b, c\},\{a, d, e\},\{a, f, g\},\{b, d, f\},\{c, e, g\}\}$ and the apex of the mitre is the point $a$.

Our main result is the following theorem.

Theorem 2.1 Let $\mathcal{S}$ be a Wilson-Schreiber system obtained from an admissible Abelian group of order greater than 9. Then $\mathcal{S}$ is universal.

To show that $\mathcal{S}$ is universal we employ a sufficient condition based on the existence of certain substructures in $\mathcal{S}$. A rooted configuration is a configuration $\mathcal{C}$ of points and 3-element blocks with one distinguished point, the root. A rooted homomorphism of $\mathcal{C}$ into $\mathcal{S}$ is a homomorphism $\mathcal{C} \rightarrow \mathcal{S}$ such that the root of $\mathcal{C}$ is mapped to a given point of $\mathcal{S}$.

The following result, based on ideas from [4] and [6], will be proved in [3].

Theorem 2.2 Let $P$ be a set of points of a Steiner triple system $\mathcal{S}$. Suppose that for every configuration $\mathcal{C}_{i} \in \mathbf{U}=\left\{\mathcal{C}_{0}, \mathcal{C}_{1}, \ldots, \mathcal{C}_{7}\right\}$ (see Fig. 1) and for every point $y \in P$ there exists a rooted homomorphism $\mathcal{C}_{i} \rightarrow \mathcal{S}$ taking the points of $\mathcal{C}_{i}$ to $P$ and the root to $y$. Then $\mathcal{S}$ is universal. 
Sketch of proof of Theorem 2.1. First observe that every non-trivial subgroup of an admissible group is admissible, and that the Wilson-Schreiber system constructed from a subgroup is a subsystem of that constructed from the whole group. By the classification of finite Abelian groups, and since $\mathbb{Z}_{2}$, $\mathbb{Z}_{3}$, and $\mathbb{Z}_{11}$ are not admissible groups, it suffices to prove the result for all admissible cyclic groups of prime order $p \geq 13$ as well as for three individual groups $\mathbb{Z}_{5} \times \mathbb{Z}_{5}, \mathbb{Z}_{5} \times \mathbb{Z}_{7}$, and $\mathbb{Z}_{7} \times \mathbb{Z}_{7}$.

Let $p \geq 13$ be a prime, and let $\mathcal{S}$ be a Wilson-Schreiber system based on $\mathbb{Z}_{p}$. To apply Theorem 2.2 , we take $P=\left(\mathbb{Z}_{p}-0\right) \cup\{\alpha, \beta\}$ and define $\mathcal{D}$ to be the partial subsystem of $\mathcal{S}$ induced by the points from $P$. For each $\mathcal{C}_{i}$ we construct two particular rooted homomorphisms $\mathcal{C}_{i} \rightarrow \mathcal{D}$, one taking the root to $\alpha$ or $\beta$ and the other taking the root to some element of $\mathbb{Z}_{p}-0$. All other rooted homomorphisms $\mathcal{C}_{i} \rightarrow \mathcal{D}$ required by Theorem 2.2 are then obtained from these two by applying automorphisms of $\mathcal{S}$. As an example, in Table 1 we display the two homomorphisms for the configuration $\mathcal{C}_{3}$. The remaining configurations, as well as the three small groups will be dealt with in [3].

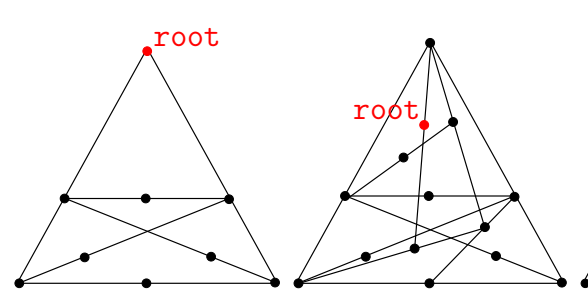

(a) $\mathcal{C}_{0}$

(b) $\mathcal{C}_{1}$

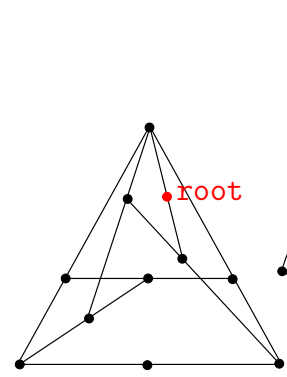

(e) $\mathcal{C}_{4}$

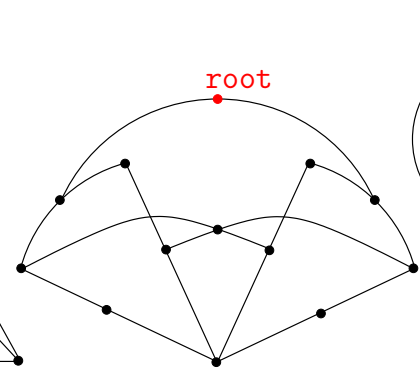

(f) $\mathcal{C}_{5}$

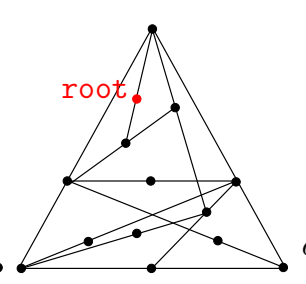

(c) $\mathcal{C}_{2}$

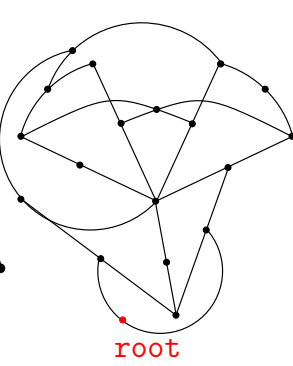

(g) $\mathcal{C}_{6}$

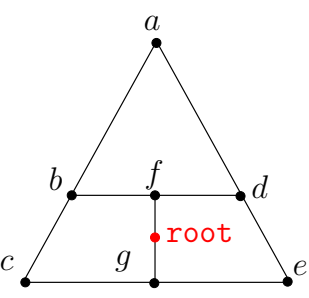

(d) $\mathcal{C}_{3}$

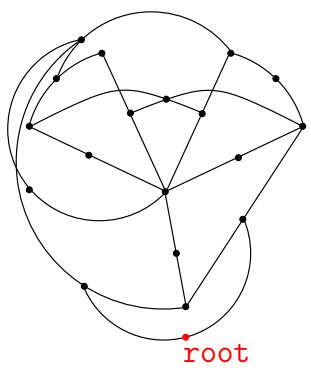

(h) $\mathcal{C}_{7}$

Fig. 1. Set of configurations $\mathbf{U}$ from Theorem 2.2 


\begin{tabular}{|c||c|c|c|c|c|c|c|c|}
\hline group & root & $a$ & $b$ & $c$ & $d$ & $e$ & $f$ & $g$ \\
\hline $\mathbb{Z}_{p}, p \geq 13$ & -2 & 1 & -3 & 2 & -4 & 3 & 7 & -5 \\
\hline & $\beta$ & 1 & 2 & -3 & -6 & 5 & 4 & -2 \\
\hline
\end{tabular}

Table 1

\section{References}

[1] Archdeacon, D., Coverings of graphs by cycles, Congr. Numer. 53 (1986), 7-14.

[2] Grannell, M. J., T. S. Griggs, M. Knor, and M. Škoviera, A Steiner triple system which colors all cubic graphs, J. Graph Theory 46 (2004), 15-24.

[3] Grannell, M. J., T. S. Griggs, E. Máčajová, and M. Škoviera, Further results on colouring cubic graphs by Steiner triple systems, in progress.

[4] Holroyd, F., and M. Škoviera, Colouring of cubic graphs by Steiner triple systems, J. Combin. Theory Ser. B 91 (2004), 57-66.

[5] Kirkman, T. P., On a problem in combinations, Cambridge and Dublin Math. J. 2 (1847), 191-204.

[6] Král', D., E. Máčajová, A. Pór, J.-S. Sereni, Characterisation results for Steiner triple systems and their application to edge-colourings of cubic graphs, Canad. J. Math., accepted.

[7] Král', D., E. Máčajová, O. Pangrác, A. Raspaud, J.-S. Sereni, and M. Škoviera, Projective, affine, and abelian colorings of cubic graphs, European J. Combin. 30 (2009), 53-69.

[8] Máčajová, E., and M. Škoviera, Fano colourings of cubic graphs and the Fulkerson Conjecture, Theoret. Comput. Sci. 349 (2005), 112-120.

[9] Pál, D., and M. Škoviera, Colouring cubic graphs by small Steiner triple systems, Graphs Combin. 23 (2007), 217-228.

[10] Schreiber, S., Covering all triples on $n$ marks by disjoint Steiner systems, J. Combin. Theory Ser. A 15 (1973), 347-350.

[11] Wilson, R. M., Some partitions of all triples into Steiner triple systems, in Hypergraph Seminar, Ohio State University 1972, Lecture Notes in Math. 411, Springer, Berlin (1974), 267-277. 